\title{
Transforming Vacant Commercial Spaces: From Localized Hotspots of Urban Shrinkage to "Smart" Co-Working Places
}

\author{
Stella Manika \\ Department of Planning and Regional Development, University of Thessaly, Volos, Greece \\ Email: smanika@uth.gr
}

How to cite this paper: Manika, S. (2020). Transforming Vacant Commercial Spaces: From Localized Hotspots of Urban Shrinkage to "Smart" Co-Working Places. Open Journal of Social Sciences, 8, 86-97. https://doi.org/10.4236/jss.2020.86009

Received: May 10, 2020

Accepted: June 2, 2020

Published: June 5, 2020

Copyright (๑) 2020 by author(s) and Scientific Research Publishing Inc. This work is licensed under the Creative Commons Attribution International License (CC BY 4.0).

http://creativecommons.org/licenses/by/4.0/

\section{(c) (i) Open Access}

\begin{abstract}
Shrinking cities (i.e., regions, cities, towns, or metropolitan areas that are facing population reductions and intense economic and social transformation) typically include an abundance of abandoned residential units, office buildings, and vacant shops. Strongly affected by the recent economic and financial crisis, Greek cities have shown strong signs of urban shrinkage with thousands of businesses closing down since 2011. These vacant properties can be reclaimed for new uses, in particular for the development of new working styles and forms of sharing economy, with the view to promote innovation and growth. Sharing economy and co-working spaces or makerspaces can play a crucial role to support social and professional interaction and promote the innovative and "smart" development of the city. This paper develops a methodological and conceptual framework to examine and document the localized hotspots of urban shrinkage that could be used as co-working spaces in Larissa, a typical medium-sized Greek city. The framework to identify the optimal localised hotspots in Larisa (i.e. vacant commercial spaces to be considered as viable co-working spaces) was based on the results of the literature review and the analysis of the respective spatial and quantitative data. These hotspots can become dynamic nodes of a network structure at a city scale contributing to the renaissance of its economic, social, and innovative profile.
\end{abstract}

\section{Keywords}

Shrinking Cities, Smart Cities, Innovation, Sharing Economy, Co-Working Spaces, Urban Renaissance, Urban Policies, Sense of Community

\section{Introduction}

Sharing economy platforms can have a crucial impact on smart cities by rede- 
signing their future (Sun et al., 2016), with an emphasis on smart governance and smart economy. These smart dimensions are two of the cumulative horizontal elements of smart cities (Caragliu et al., 2011; Gori et al., 2015). However, despite the expansion of sharing economy platforms at multiple scales and their proven benefits, in practice, their application in smart cities' planning and policies is currently underutilized (Fiorentino \& Bartolucci, 2019). Based on a theoretical reflection on the interrelation between the city and its common high-density development, cities are naturally structured as sharing economies "with consumption involving access to shared resources" (Sun et al., 2016; Agyeman \& McLaren, 2014).

Vacant commercial or residential spaces are often considered as an obstacle to the structure and viability of the urban space. Therefore, any attempt to re-define their role in urban reality can be beneficial, especially when it is combined with a broader "smart" strategic planning at municipal level. However, the literature that focuses on the development capabilities of coworking spaces in shrinking areas is relatively limited.

Abandoned residential units or vacant shops, especially in shrinking cities, can become the key pillar of city efforts to create new opportunities, pose new challenges, promote resource usage efficiency, and "innovation at the grassroots level” (Wang \& Loo, 2017). Considering the sharp increase of vacant spaces, due to the recent economic crisis in Greece, and the limited experience and application of co-working spaces, Greek cities can present a fertile environment for the development of sharing economy platforms.

\section{Literature Review}

The concepts of "smart city", "intelligent city", "knowledge city" (Anttiroiko et al., 2014) or "digital city" (Couclelis, 2004) are broad ideas that include many aspects of urban life, as a response to the city's problems (such as increased traffic jams, carbon dioxide or greenhouse gas emissions, etc.). Several features can determine a city as "smart", including among others, broadband connectivity, communication infrastructure, knowledge workforce, and digital inclusion (Sun et al., 2016). Moreover, a common characteristic of all smart cities is the transformation of their modus operandi by using information and communication technology (ICT), achieving to support communal sharing (Agyeman \& McLaren, 2014). The sharing economy is an evolving economic practice that aims to promote resource efficiency and collaboration of individuals and consumers, via sharing the use of idle assets and reducing transaction costs (Gori et al., 2015). In the sharing economy, governances reclaim new perspectives (as an illustration, blockchain applications) (Fiorentino \& Bartolucci, 2019). In addition, individuals are also encouraged to share spare goods with others and offer underutilized assets, regardless of whether such assets are monetized or not, such as short-term rentals of their vehicles, or spare rooms in their houses. Hence, consumers can benefit from greater variety and lower costs, compared to the alter- 
natives offered by traditional industry, having the flexibility to access goods and services only for as long as they are needed.

The trend of sharing economy model, based on a sharing phenomenon "as old as humankind" (Belk, 2014), has been increasingly adopted by individuals and businesses which encourage the use of co-working spaces. These co-working spaces, featured as "microclusters" (Capdevila, 2014), can be within one company or space where many individuals (startups, freelancers) work together towards their independent goals to reduce the rent cost and office expenses, leading these spaces to be characterized as "creative and energetics" (Fuzi, 2015). Apart from the proven benefit of cost efficiency, co-working spaces have several other significant advantages. In particular, they may stimulate social interactions and sense of community, networking opportunities, boost productivity and creativity with knowledge and idea exchanges and provide a uniquely innovative environment that offers the opportunity to collaborate with like-minded professionals in different industries. Therefore, from the sharing economy and co-working places' perspective, smart cities can benefit from the availability of numerous abandoned or empty buildings in shrinking cities, as target areas of "urban renewal" policies (Ryan, 2008).

Research conducted in several European cities has demonstrated that the main drivers for the evolution and development of co-working spaces constitute the increasing need for professional flexibility, the encouragement of healthy work-life balance (e.g. spatial proximity to the coworkers' homes) and the resulting economic benefits (Kojo \& Nenonen, 2017). Several researches have concluded that the most efficient co-working spaces are located in urban units that present the following characteristics:

1) Increased density of commercial activities (Mariotti et al., 2017), mainly in medium or large-sized cities (Fuzi, 2015);

2) Sufficient accessibility by car or public transport, as part of a broader urban traffic system (Deskmag, 2012; Weijs-Perrée et al., 2019; Capdevila, 2014);

3) Close distance from research institutes or universities (Mariotti et al., 2017) and public services (Wang \& Loo, 2017);

4) Located in the city centre, preferably on the inner ring road. However, the creation of co-working spaces in the central business district of a city could be discouraged due to the high property price in these areas (Wang \& Loo, 2017);

5) Aligned with the co-worker's specific needs and preferences to maximise the users' satisfaction (Weijs-Perrée et al., 2019).

Despite the fact that the concepts of smart cities and co-working spaces are at the centre of inter-disciplinary debates (Kojo \& Nenonen, 2017), several critical urban questions still remain unanswered. The most characteristic one include "which is the appropriate location pattern of co-working spaces and makerspaces in order to proliferate their benefits at multiple scales". This paper examines the aforementioned parameters by analyzing and identifying the optimal locations for co-working spaces in a medium-sized Greek city. 


\section{Methodology, Data, Sources and, Study Area}

The study was conducted in the city of Larissa (Greece), that was selected due to its following significant characteristics:

1) The morphological pattern of the city, i.e. the urban fabric of Larissa is characterized by the typical morphology (streets, squares, urban blocks) of modern Greek cities, as a result of post-war housing development patterns in Greece;

2) Larissa is a medium-sized Greek city, with a permanent population equal to 145,000 according to the 2011 census. Larissa largely demonstrates the average characteristics observed for the majority of Greek cities, apart from Athens, Thessaloniki, and Piraeus;

3) Larissa suffers from lack of a specific economic identity (such as tourism for Greek islands);

4) Due to the recent economic crisis, Larissa is a typical Greek city in terms of shrinking signs, exhibiting thousands of closing enterprises, a series of vacant shops (even in previously high commercial streets), abandoned office buildings, and a dramatic decline in construction activity. At the same time, similarly the majority of Greek cities, Larissa exhibits spatial rearrangements of the population; relocation of households in other neighborhoods in search of lower living costs (Gospodini, 2012);

5) Several public universities (such as University of Thessaly) and private research institutions are located therein;

6) Similar to other medium-sized Greek cities, the initiatives and efforts to create co-working spaces or makerspaces in Larissa are limited so far.

Given its particular characteristics and the absence of the required initiatives towards the creation of co-working spaces therein, Larissa can be considered as an appropriate example of a city to apply relevant strategies and urban policy actions with the aim to adopt sharing economy pattern.

The underlying data used in the context of the present research were sourced from the Chamber of Commerce located in Larissa. In order to identify the impact of economic and spatial shrinkage in Larissa, the commercial activity data concern companies operating therein during the period from 01/01/2008 to 31/12/2014 (monitored monthly). The year of 2008 was used as the starting point for the survey given that the effects of the economic crisis in the city's commercial landscape were not visible at that stage. Hence, this year's picture (economically and demographically) can be the basis of time-tracking and efficient comparison. From the primary data set, were selected businesses that 1) operated within Larissa and 2) belonged to the private sector having a specific legal status (individual, public limited, limited partnership, private equity, etc.). The year 2014 represents the end of the first phase of economic and spatial shrinkage in Larissa, showing the preliminary signs of stabilization and interruption of this phenomenon.

The categorization of land use data was based on NACE Rev. 1 "Statistical Classification of Economic Activities in the European Community" in force 
since 2008. The database created the appropriate digital backgrounds (road axis and urban blocks) and point databases. The followed procedure was geocoding, whereby the desired cartographic data was produced using information describing sites such as postal addresses and address locators. Through this process, most of the businesses immediately acquired spatial reporting. Other businesses were manually matched with the help of location programs (such as Google Maps and Google Earth).

The research process starts with the calculation of the ratio between unoccupied and occupied business premises per building block during the aforementioned study period (2008-2014). At the first stage, the research identifies urban units in adjacent urban blocks in Larissa with similar characteristics that present a high ratio of unoccupied/occupied buildings, indicating their vulnerability to spatial and economic shrinkage. The research continues with the identification of areas within Larissa that present an increased frequency - rotation of new recordings over the period under consideration. Following the identification of urban units vulnerable to urban shrinkage and areas with increased density, the analysis detects the local points that could constitute hotspots of urban shrinkage, which can be the optimal location for co-working spaces in Larissa. Based on this analysis, the research identifies not only the spatial distribution of the ratio per building block but also the spatial patterns (spatial units consisting of urban blocks). Therefore, the research performs a spatial analysis at different scales (city areas and selected urban units) and a different typological form (urban blocks and points). The identified urban units with a high ratio between unoccupied and occupied business premises and an adequate frequency - rotation of new recordings constitute the optimal location for co-working spaces in Larissa.

\section{Step 1: Identification of Areas Vulnerable to Urban Shrinkage}

The calculation of the ratio between unoccupied and occupied business premises does not demonstrate significant variation during the study period. The application of this ratio per building block and its spatial performance as described in Figure 1 and Figure 2 present the following results of economic and spatial urban shrinkage in the respective areas of Larissa. Both maps reveal that in 2008 and 2014, the urban units in Larissa with the highest percentage of the ratio between unoccupied and occupied business premises are located on less central areas (Philipoupolis, Neraida, Laxanokipoi, Ampelokipoi). Furthermore, in the central districts located within the internal ring road, there is a plethora of urban blocks corresponding to small and medium values of the ratio. The difference between the maps for 2008 and 2014 is that in the latter the index prices observed are higher.

Therefore, the research observes that the less central districts of the study area present higher rates of urban contraction, making them vulnerable to the phenomenon of urban shrinkage. For this reason, it is important to identify the local 


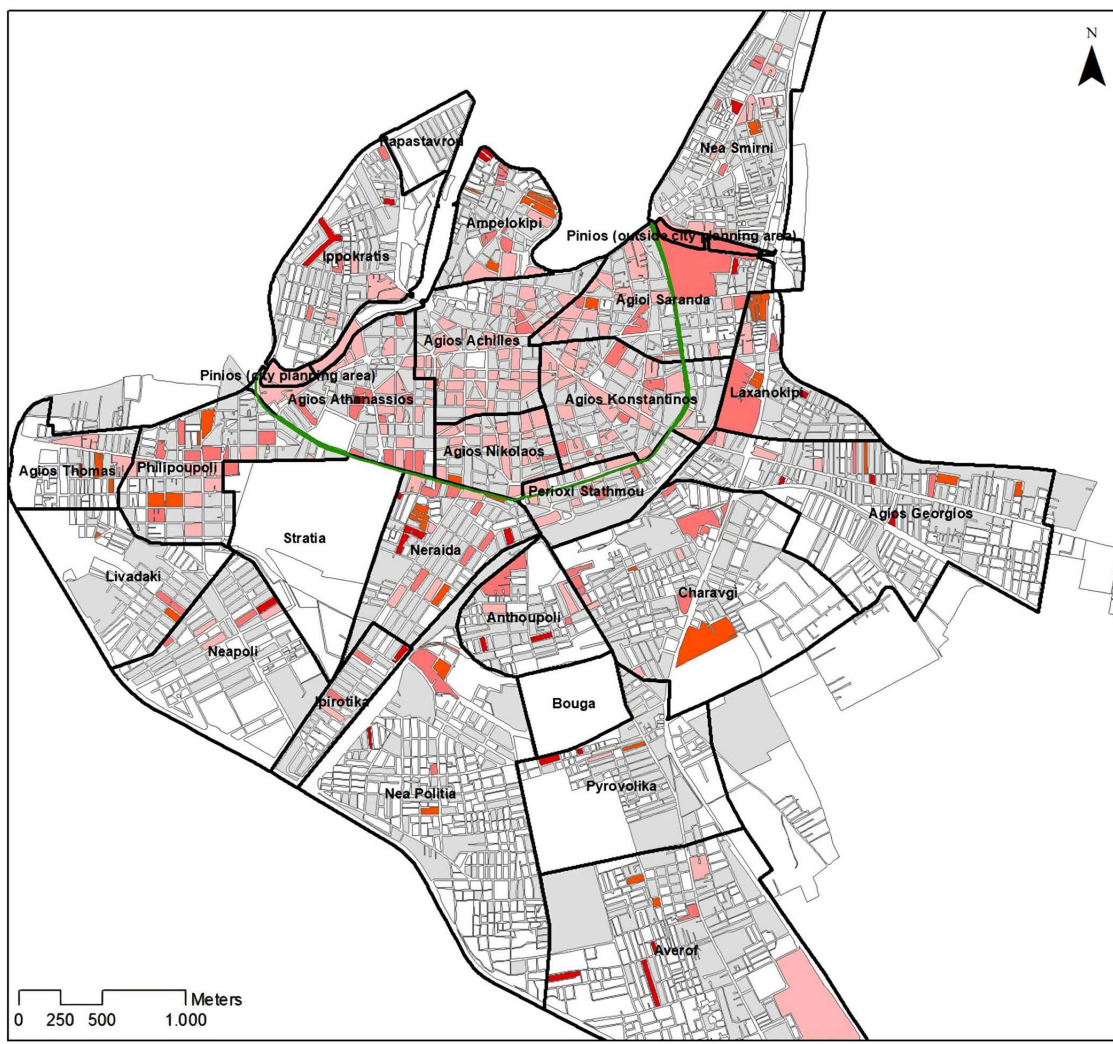

Ratio between unoccupied and occupied business premises (2008)

\section{Rates \%}

$0-5$

$\square-20$

$20-40$

$40-60$

$>60$

$\square$ Urban Units

No business premises

__ Internal Ring Road

Source: Larissa Chamber of Commerce (own processing)

Figure 1. Ratio between unoccupied and occupied business premises, 2008.
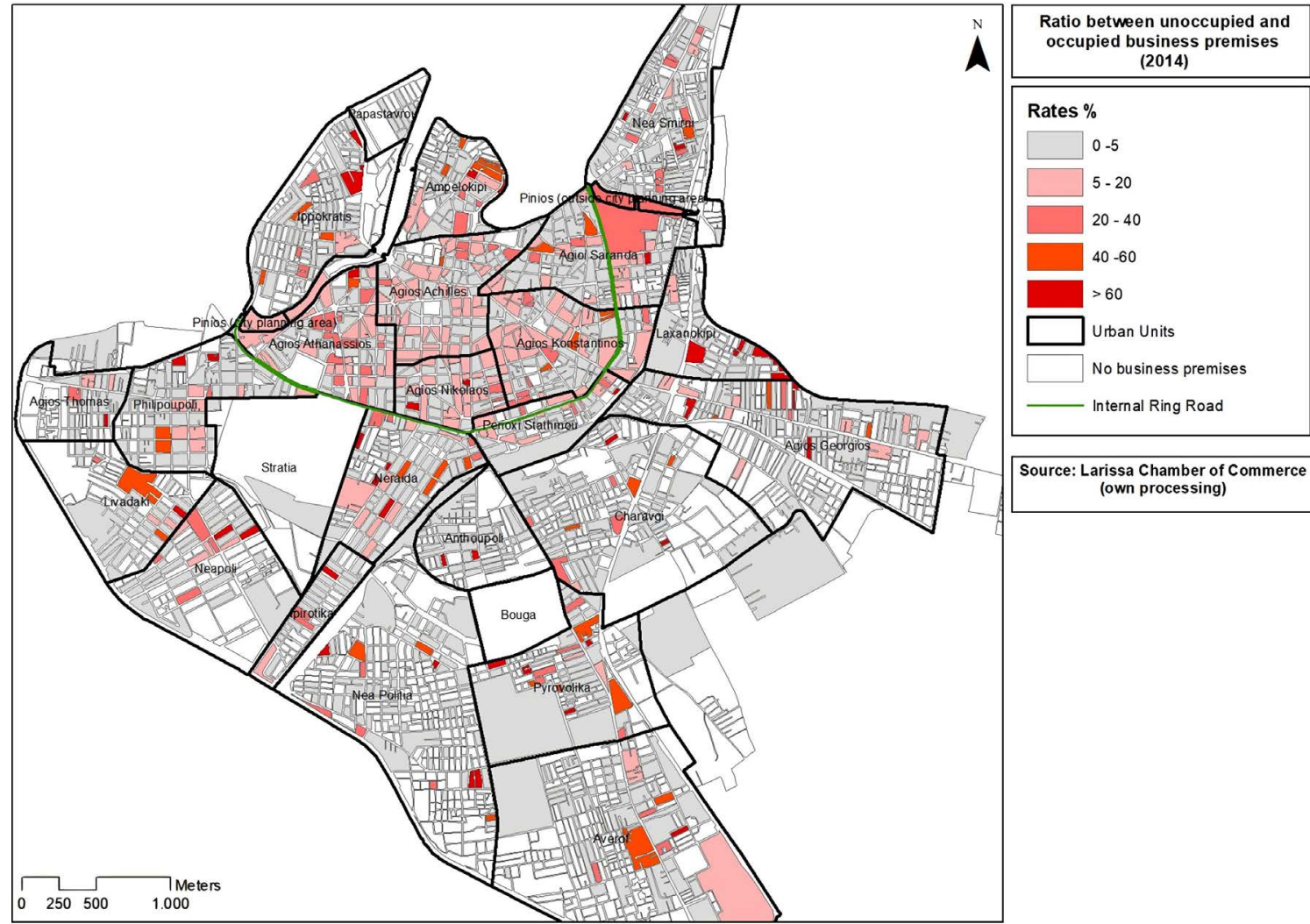

Source: Larissa Chamber of Commerce (own processing)

Figure 2. Ratio between unoccupied and occupied business premises, 2014. 
points that present a medium/high frequency - rotation of new recordings, within the vulnerable urban units, over the period under consideration.

\section{Step 2: Identification of Localized Hotspots}

In order to identify the most beneficial location for co-working spaces in Larissa, the study continues with the further identification of localized hotspots of urban shrinkage in the urban units of Larissa. Localized hotspots of urban shrinkage are points (business premises) that present a medium/high frequency - rotation of new recordings over the period under consideration. The reported local points to the Chamber of Commerce in Larissa, from 2008 to 2014, were examined. The number of new registrations completed for each local point in Larissa during the study period has demonstrated (Figure 3 ) the following:

1) Local points of increased intensity of urban shrinkage through the frequent rotation of new registrations $(8-12)$ or alternation of short-lived businesses in these areas;

2) Local points of lower intensity of urban shrinkage due to the limited number of new registrations (4 - 8); and

3) Local points that present a stable commercial activity (2 - 4 new registrations).
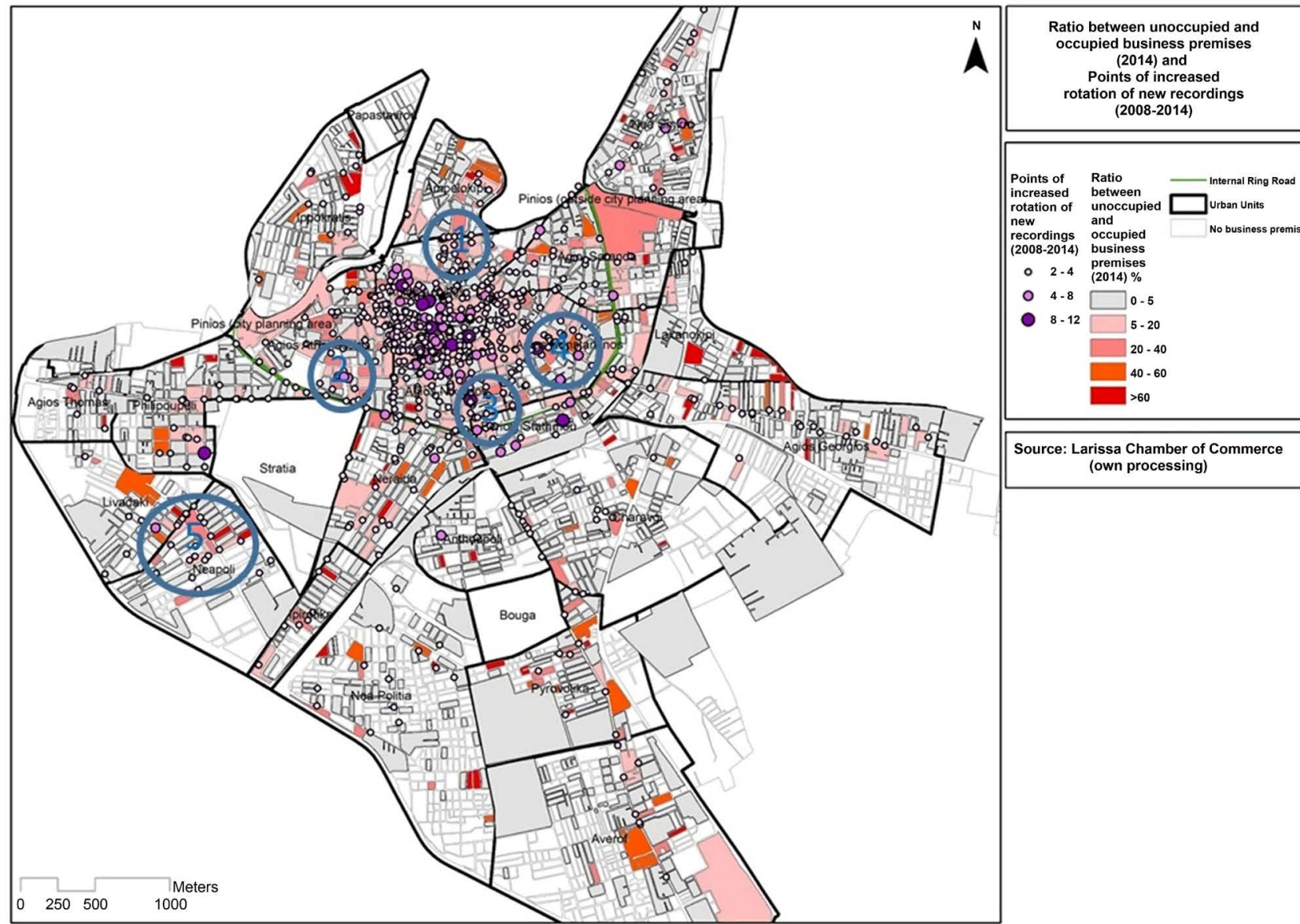

Source: Larissa Chamber of Commerce (own processing)

Figure 3. Spatial results of the application of the two steps. 
It is observed that the areas, which include the points with an increased frequency - rotation of new recordings, are concentrated in the business centre (Figure 3). Therefore, these local points constitute the business headquarters of start-ups or companies that have moved to the city centre from another less privileged location.

The identification of local points with a very high frequency - rotation of new recordings in the city centre, confirmed that, even though the professional spaces of the central districts can remain empty mainly due to the high rent, they can get "refilled" very quickly. Their resilience, despite the significant impact of the economic crisis, is based on their privileged position in the established shopping centre.

At the same time, there is an increased concentration of points mainly of medium frequency - rotation of new recordings especially in areas located around the established business centre and inside the inner ring. These points can be considered as lower-performing or even "cursed" locations that do not contribute to the economic growth of the city (Manika, 2018). Combining the spatial results of the application of the two steps, we can identify some areas that present two main cumulative characteristics: 1) urban blocks of an increased percentage of empty stores, and 2) medium to high rotation index. These areas, as shown in Figure 3, are located around the established business centre and in close proximity to the inner ring (indicatively given in circles $1-4$ ) or in less central urban planning units (circle 5).

Based on the principles of co-working spaces and their ability to reactivate the economic and social dynamics of a region, we propose the creation and promotion of co-working spaces in empty commercial spaces within the areas selected by the spatial analysis.

In particular, within the selected areas around the city centre and in close proximity to the inner ring, it is proposed to create large co-working spaces. These areas include suitable vacant properties for such usage, for example, inactive hotels, previous cinemas, etc. In many cases, several of these properties are even state-owned, which could allow cities to explore further income possibilities and hence promote co-working related activities.

However, due to the increased costs and required investment funds for establishing large co-working spaces, the option of less central districts can be an alternative. The identified district in the less central unit in Larissa (circle 5) has a plethora of closed small-sized shops, which could be utilized for creating smaller co-working spaces. In case, such properties are adjacent and fulfill the required conditions, they could create opportunities for synergies and be joined together to constitute larger co-working spaces, in most cases at a relevantly lower cost.

In addition, the proposed options of large or small co-working spaces in the identified local points satisfy the criteria presented in the bibliography, regarding the coverage by the existing network infrastructure and their proximity to educational institutions, public services, etc. In particular, the University is located near circle 5 . 
The co-working spaces in the proposed local points can be united by forming nodes of a network that promotes the sharing economy and encourages creative or innovative industries. Given that co-working spaces are more likely to thrive when they are located in central districts, as it is supported by the prevailing literature, the constructive collaboration via networking and knowledge sharing between co-working spaces in central districts with those in less central areas can improve their viability. These urban units can become local hotspots creating networks that will essentially function as network nodes, increasing their efficiency. The use of smart applications and smart service platforms with an emphasis on sharing economy and co-working places in these hotspots should be further encouraged.

\section{Results}

Based on the analysis above, it is concluded that the economic crisis had several and different impacts on the various scales of the urban fabric of Larissa. To investigate which areas and local points are mostly affected, the ratio between unoccupied and occupied business premises was calculated for a specific study period. The spatial analysis (step 1) showed that the less central districts of the study area and the areas around the established business centre demonstrate higher and medium rates of vacant lots, thus making them vulnerable to the phenomenon of urban shrinkage.

In order to identify the optimal location for co-working spaces in Larissa, the study continued with the further identification of local points with a medium/high frequency - rotation of new registrations in the various urban units (step 2). Combining the spatial results of the application of the two steps, areas that present two main cumulative characteristics, 1) urban blocks of an increased percentage of empty stores, and 2) medium to high rotation index, identified. These areas are located around the established business centre and in close proximity to the inner ring or in less central urban planning units. The hotspots located in urban blocks of an increased percentage of empty stores and an adequate frequency - rotation of new recordings constitute the optimal location for co-working spaces in Larissa.

Furthermore, considering the role of co-working spaces as a lever of development and innovation, creating co-working spaces (smaller or larger, depending on the availability of empty stores) in the identified local hotspots would be an important step to achieve a smarter city approach. Networking among co-working spaces can increase their overall viability. The transformation of all vacant spaces into co-working spaces is not a realistic option. However, exploring the highlighted local points as potential co-working spaces to reactivate them, would have multiple benefits for the social and economic development of the wider region.

\section{Conclusion}

With the view to achieving the "intelligence" of a city, it is important that the 
policies adopted refer to both urban scale and local scale. In order to increase the effectiveness and efficiency of sharing economy initiatives, it is required to first recognize the most vulnerable urban areas and then move forward with smart solutions by taking into consideration the individual characteristics of each area. The adoption of sharing economy and co-working spaces can lead to the social and economic development of the wider region and eventually to a new more "smarter" approach regarding urban planning. Furthermore, similar considerations can be applied in the cases of equivalent sized Greek cities with comparable characteristics.

Strongly affected by the recent economic crisis, Larissa has to use the tactics of "3Re Strategy" (repurpose-renew-reconnect) (Revitalization News, 2020) in order to revitalize neighbourhoods and boost innovation, urban growth, and citizen well-being. The creation of co-working spaces can be one step towards this direction.

Despite the recognized value of co-working spaces, Larissa hesitates to provide State-owned or Municipal-owned unoccupied buildings for the use of co-working spaces. The most common barriers for cities that hinder their capacity to encourage innovative practices are the inherent risk aversion of the public sector and their limited capacity related to new technologies and market developments (OECD, 2017).

Structuring partnerships with the private sector should be also encouraged. Cities can engage in a constructive collaboration with the private sector, with the aim to achieve common objectives and benefit from knowledge sharing and exchange of best practices in the private sector. Such an approach will increase awareness and bring a different set of stakeholders to the table, creating a strong alliance for change.

\section{Acknowledgements}

This research is co-financed by Greece and the European Union (European Social Fund - ESF) through the Operational Programme «Human Resources Development, Education and Lifelong Learning» in the context of the project "Reinforcement of Postdoctoral Researchers - 2nd Cycle" (MIS-5033021), implemented by the State Scholarships Foundation (IKY).

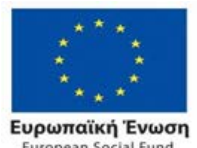

Operational Programme Human Resources Development, Education and Lifelong Learning

co-financed by Greece and the European Union

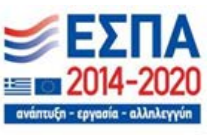

\section{Conflicts of Interest}

The author declares no conflicts of interest regarding the publication of this paper.

\section{References}

Agyeman, J., \& McLaren, D. (2014). “Smart Cities” Should Mean "Sharing Cities". 
https://time.com/3446050/smart-cities-should-mean-sharing-cities/

Anttiroiko, A. V., Valkama, P., \& Bailey, S. J. (2014). Smart Cities in the New Service Economy: Building Platforms for Smart Services. AI and Society, 29, 323-334.

https://doi.org/10.1007/s00146-013-0464-0

Belk, R. (2014). You Are What You Can Access: Sharing and Collaborative Consumption Online. Journal of Business Research, 67, 1595-1600. https://doi.org/10.1016/j.jbusres.2013.10.001

Capdevila, I. (2014). Knowledge Dynamics in Localized Communities: Coworking Spaces as Microclusters. SSRN Electronic Journal, 19, No. 3. https://doi.org/10.2139/ssrn.2502813

Caragliu, A., del Bo, C., \& Nijkamp, P. (2011). Smart Cities in Europe. Journal of Urban Technology, 18, 65-82. https://doi.org/10.1080/10630732.2011.601117

Couclelis, H. (2004). The Construction of the Digital City. Environment and Planning B: Planning and Design, 31, 5-19. https://doi.org/10.1068/b1299

Deskmag (2012). The 2nd Annual Coworking Survey. http://reseau.fing.org/file/download/128857

Fiorentino, S., \& Bartolucci, S. (2019). New Governance Perspectives on the Sharing Economy. A Blockchain Application for the "Smart" Management of Co-Working Spaces with a Return for Local Authorities. SSRN Electronic Journal. https://doi.org/10.2139/ssrn.3478870

Fuzi, A. (2015). Co-Working Spaces for Promoting Entrepreneurship in Sparse Regions: The Case of South Wales. Regional Studies, Regional Science, 2, 462-469. https://doi.org/10.1080/21681376.2015.1072053

Gori, P., Parcu, P. L., \& Stasi, M. L. (2015). Smart Cities and Sharing Economy. SSRN Electronic Journal. https://doi.org/10.2139/ssrn.2706603

Gospodini, A. (2012). Economic Crisis and the Shrinking Greek Cities. 1st International Conference on Architecture and Urban Design, Epoca University, 19-21 April 2012. http://dspace.epoka.edu.al/bitstream/handle/1/260/127-567-1-PB.pdf?sequence=1\&isAl lowed $=y$

Kojo, I., \& Nenonen, S. (2017). Evolution of Co-Working Places: Drivers and Possibilities. Intelligent Buildings International, 9, 164-175. https://doi.org/10.1080/17508975.2014.987640

Manika, S. (2018). Economic Crisis, Shrinking Greek Cities, and Urban Regeneration Policies. Volos: University of Thessaly.

Mariotti, I., Pacchi, C., \& Di Vita, S. (2017). Co-Working Spaces in Milan: Location Patterns and Urban Effects. Journal of Urban Technology, 24, 47-66. https://doi.org/10.1080/10630732.2017.1311556

OECD (2017). Public Procurement for Innovation: Good Practices and Strategies, OECD Public Governance Reviews. Paris: OECD Publishing.

Revitalization News (2020). Word Urban Campaign. https://www.worldurbancampaign.org/revitalization-news

Ryan, B. D. (2008). The Restructuring of Detroit: City Block Form Change in a Shrinking City, 1900-2000. Urban Design International, 13, 156-168. https://doi.org/10.1057/udi.2008.21

Sun, J., Yan, J., \& Zhang, K. Z. K. (2016). Blockchain-Based Sharing Services: What Blockchain Technology Can Contribute to Smart Cities. Financial Innovation, 2, 26. https://doi.org/10.1186/s40854-016-0040-y

Wang, B., \& Loo, B. P. Y. (2017). Hubs of Internet Entrepreneurs: The Emergence of 
Co-working Offices in Shanghai, China. Journal of Urban Technology, 24, 67-84. https://doi.org/10.1080/10630732.2017.1285124

Weijs-Perrée, M., van de Koevering, J., Appel-Meulenbroek, R., \& Arentze, T. (2019). Analysing User Preferences for Co-Working Space Characteristics. Building Research and Information, 47, 534-548. https://doi.org/10.1080/09613218.2018.1463750 